\title{
Multi-scale pressure-balanced structures in the solar wind observed by WIND
}

\author{
YAO Shuo $^{1 *}$, TU ChuanYi ${ }^{2}$, HE JianSen ${ }^{2}$, WEI WenBo ${ }^{1} \&$ MENG XiaoHong ${ }^{1}$ \\ ${ }^{1}$ School of Geophysics and Information Technology, China University of Geosciences (Beijing), Beijing 100083, China; \\ ${ }^{2}$ School of Earth and Space Sciences, Peking University, Beijing 100871, China
}

Received October 5, 2011; accepted November 18, 2011; published online February 21, 2012

\begin{abstract}
This work detects multi-scale, from hour to seconds, pressure-balanced structures (PBSs) in the solar wind based on the anticorrelation between the plasma thermal pressure and the magnetic pressure measured by WIND at 1 AU on April 5th, 2001. In our former research based on Cluster measurements, we showed the anti-correlation between the electron density and the magnetic field strength in multi-scales, and we supposed these structures may be pressure-balanced structures. Thus, in this work we aim to prove our speculation by the direct evidence on pressure measurements. Different from our previous work, we apply the WIND measurements this time, for they have both the magnetic pressure and the plasma pressure which Cluster could not offer. We use the wavelet cross-coherence method to analyze the correlation between the plasma pressure $\left(P_{t h}\right)$ and the magnetic pressure $\left(P_{B}\right)$, and also the electron density $\left(N_{e}\right)$ and the magnetic field strength $(B)$ on various scales. We observe the anti-correlation between $P_{t h}$ and $P_{B}$ distributed at different temporal scales ranging from $1000 \mathrm{~s}$ down to $10 \mathrm{~s}$. This result directly indicates the existence of pressurebalanced structures (PBSs) with different sizes in the solar wind. Further, We compare the wavelet cross correlation spectrum of $P_{t h}-P_{B}$ and $N_{e}-B$. We notice that the two spectra are similar in general. Thus this result confirms that the relation between $P_{t h}-P_{B}$ and $N_{e}-B$ are consistent with each other in the PBSs we study. Moreover, we compare the power spectrum density (PSD) of relative $N_{e}$ fluctuation with our previous work based on Cluster measurements. The two spectra show similar trend with Komolgorov's $-5 / 3$ as their slopes. This may imply the similarity of the structures observed by both WIND and Cluster spacecrafts. Finally, we discuss the possible formation mechanisms for these multi-scale pressure-balanced structures. Our result is important to support the existence of multi-scale PBSs from one-hour scale down to one-minute, and is helpful to understand the role of compressive fluctuation in the solar wind turbulence dominated by Alfvénic cascading.
\end{abstract}

solar wind, structures, turbulence

Citation: Yao S, Tu C Y, He J S, et al. Multi-scale pressure-balanced structures in the solar wind observed by WIND. Chin Sci Bull, 2012, 57: 1421-1428, doi: 10.1007/s11434-011-4966-1

Pressure-balanced structures (PBSs) typically are defined by the anti-correlation between the fluctuations of the thermal pressure $\left(P_{t h}\right)$ and magnetic pressure $\left(P_{B}\right)$ with the total pressure staying steady. Because of the common observations in the solar wind, the PBSs are believed to be the major ingredients of the solar wind turbulence and one of the most prominent compressive fluctuations in the solar wind (see a review [1] and references therein).

The first PBS was identified in the solar wind [2], accord-

*Corresponding author (email: yaoshuo@cugb.edu.cn) ing to the anti-correlation between the plasma thermal pressure and the magnetic pressure, when the total pressure did not change. Besides, Burlaga and Ogilvie [2] reported that $P_{t h}$ and $P_{B}$ were negatively correlated on one-hour scale, indicating the existence of PBSs on one-hour scale. Thieme et al. [3] found similar evidence for PBSs in the eclipticplane solar wind and suggested them originating from corona. Marsch and Tu [4,5] analyzed the correlations between different pairs of parameters like the density, the magnetic field magnitude and so on by Helios data, indicating the $P_{t h}-P_{B}$ and $n-B$ have the same trend of correlation in PBSs. Thus, 
Tu et al. [5] suggested a model invoking a superposition of PBSs and perpendicular fast magnetosonic waves, which could qualitatively explain the compressive fluctuations observed in the solar wind. In the polar solar wind observed by Ulysses spacecraft, the PBSs were also identified from the anti-correlation of $P_{t h}$ and $P_{B}$, and found to exist at scales from half a day down to one hour [6-8].

Besides, the solar wind pressure is believed to play a key role in the interaction between the solar wind and the earth magnetosphere. The Dst index of the magnetic storm was reported to have positive relation with the solar wind dynamic pressure [9]. In another aspect, Wei et al. [10] indicated that the solar wind with high dynamic pressure and high velocity may help transport more energy into the magnetosphere. Zong et al. [11] suggested small solar wind pressure pulses or small dynamic pressure changes could impact the radiation belt dynamics. Thus, it is necessary and important to study the pressure variation, for example the pressurebalanced structures in the solar wind.

Since the anti-correlation between fluctuations of the plasma density $n$ and the magnetic field magnitude $B$ was often observed in association with PBSs $[5,8,12]$ in the ecliptic plane, the $n-B$ anti-correlation may be used to identify the PBSs at 1 AU. Currently, the distribution of solar wind electrons was reported to have relation with the moon magnetic field in the near-moon space [13]. Above all, it is also interesting to study the relation between the electron density variation and that of the magnetic field in the solar wind.

Previously, Kellogg et al. [14] found possible PBSs with scales of seconds from the discrete anti-correlation of $N_{e}$ (electron density) and $B$ in the solar wind. Recently, Howes et al. [15] found the fluctuation of proton density was anti-correlated against the fluctuation of magnetic field's parallel component from their statistical study on WIND measurements at $1 \mathrm{AU}$. And they suggested this anti-correlation indicating the solar wind turbulence prone to being controlled by kinetic Alfvén wave rather than slow whistler wave. Yao et al. [16], based on the Cluster measurements, for the first time observed anti-correlation between $N_{e}$ and $B$ at various scales from one hour to 10 seconds in the same solar wind period, indicating the possible existence of PBSs. However, since the Cluster measurements could not offer convincing electron temperature and thus the plasma thermal pressure, a direct evidence from definition to support the existence of these multi-scale PBSs is still in a lack. Different from Cluster, WIND could provide the magnetic pressure and the thermal pressure of both protons and electrons. So we decided to use WIND measurements to carry out the pressure analysis on pressure-balanced structures.

Therefore, our research is focused on the relation of the plasma thermal (kinetic) pressure fluctuations and the magnetic pressure fluctuations. This work is to directly determine if the pressure-balanced structures from 1-h scale down to 10s scale are continuously embedded in the solar wind. Further, we compare the results from Cluster measurements [16] with that from WIND measurements. Finally, we discuss the probable producing mechanisms of the detected small scale PBSs.

\section{Observations}

\subsection{Instrument and data}

In this work, we apply the data measured by WIND spacecraft published by NASA's CDA web. The WIND spacecraft was launched in the year 1994 and it was designed to measure the plasmas and magnetic field in the solar wind and in the earth magnetosphere [17]. In the period we study, from April 5th to April 6th, WIND was in the solar wind and about $200 R_{E}$ away from the earth bow shock on $Y$ direction in the GSE (Geocentric Solar Ecliptic) coordinate. In our research, we obtain the proton density $\left(N_{p}\right)$, the proton temperature $\left(T_{p}\right)$ and the solar wind velocity $(V)$ from the instrument Three Dimensional Plasma and Energetic Particles Investigation (3DP) onboard WIND [18]. We obtain the electron density $\left(N_{e}\right)$, electron temperature $\left(T_{e}\right)$ from the instrument Solar Wind Experiment (SWE) on WIND [19]. We obtain the magnetic field magnitude $(B)$ and its GSE components from the instrument Magnetic Field Investigation (MFI) on WIND [20]. The magnetic field data and proton data have a time resolution of $3 \mathrm{~s}$, while the electron data has a time resolution of 6-12 s. Because of the difference in the time resolution, we use linear interpolation on the electron data to match the other two kinds of data in analyzing process. All the data we used are in the GSE coordinate. Besides, we use the proton temperature and density to calculate the proton thermal pressure $\left(P_{t h}(p r o)\right)$ and the electron temperature and density for the electron thermal pressure $\left(P_{t h}(e)\right)$. We take the sum of the proton and electron thermal pressure as the plasma thermal pressure. The total pressure in our analysis is the sum of the plasma thermal pressure and the magnetic pressure. In order to depress the random noise in the power spectrum density (PSD), we apply the multitaper method [21,22].

To study the correlation between $P_{t h}-P_{B}$ and $N_{e}-B$, we introduce the Morlet wavelet cross-coherence method, which was developed in [23]. Since we need to discriminate the positive correlation and negative correlation, we show the spectrum of the cross coherence coefficient together with the spectrum of the absolute phase angle. The detailed description of the spectra of coherence coefficient and coherent phase angle was stated in [16].

\subsection{Observation}

We choose a solar wind period measured by WIND spacecraft at $1 \mathrm{AU}$, which begins from 19:00 April 5th, 2001 and ends at 04:00 April 6th, 2001. In this period, the proton density is between 3 and $8 \mathrm{~cm}^{-3}$, with the proton temperature around $0.2-0.3 \mathrm{MK}$. The solar wind velocity is about $550 \mathrm{~km} / \mathrm{s}$ mainly on $x$ direction of GSE coordinate. The electron density varies between 3 and $8 \mathrm{~cm}^{-3}$, and the electron temperature stays steady around $0.17 \mathrm{MK}$. All the above are 


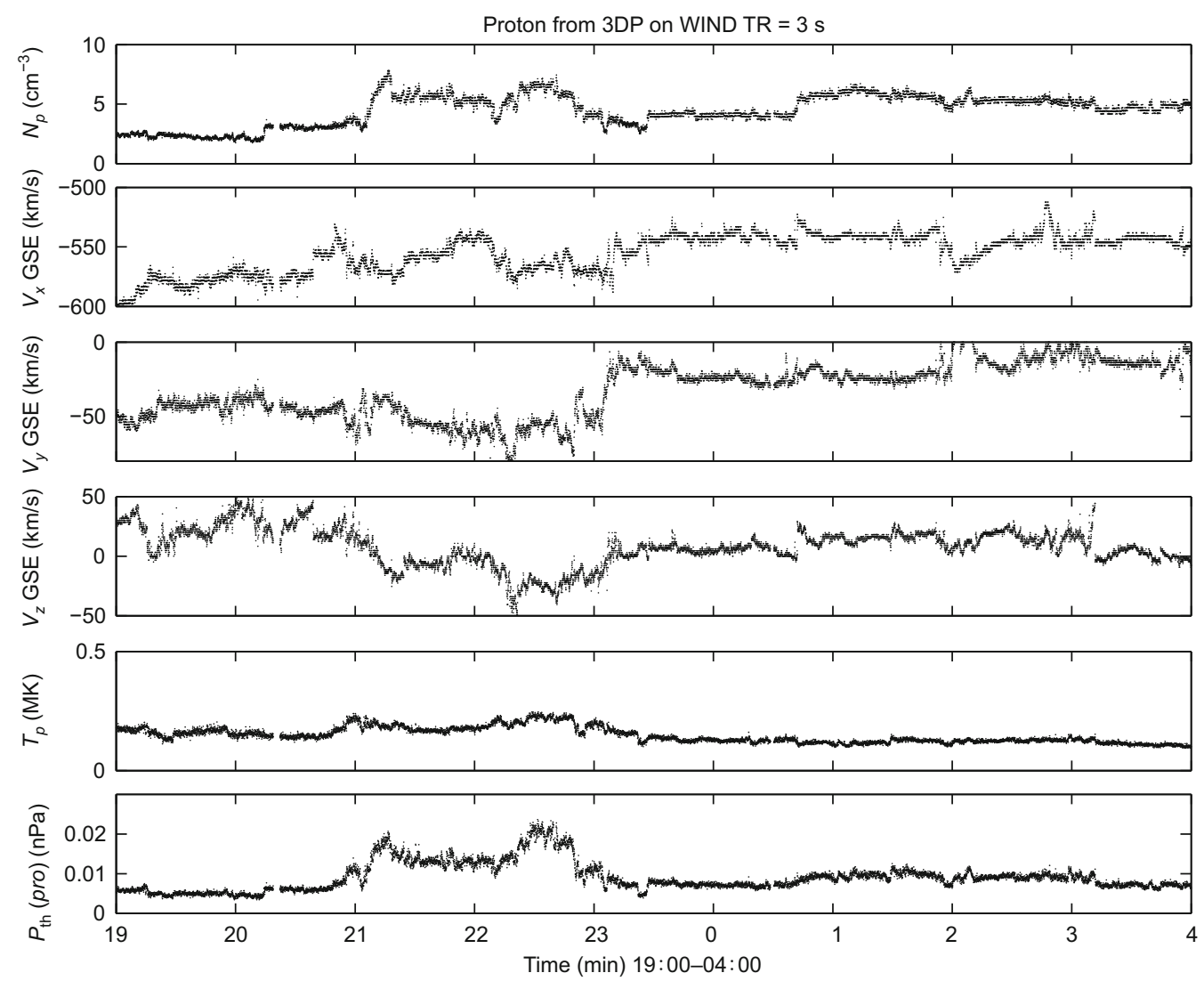

Figure 1 An overview of the measurements from 3DP on WIND spacecraft between 19:00 April 5th and 04:00 April 6th, 2001 UT. The five panels show the proton number density, the solar wind velocity magnitude and the $x, y, z$ components in GSE coordinates. The last two panels shows the proton temperature and its thermal pressure. The measurements are shown in the figure as data points.
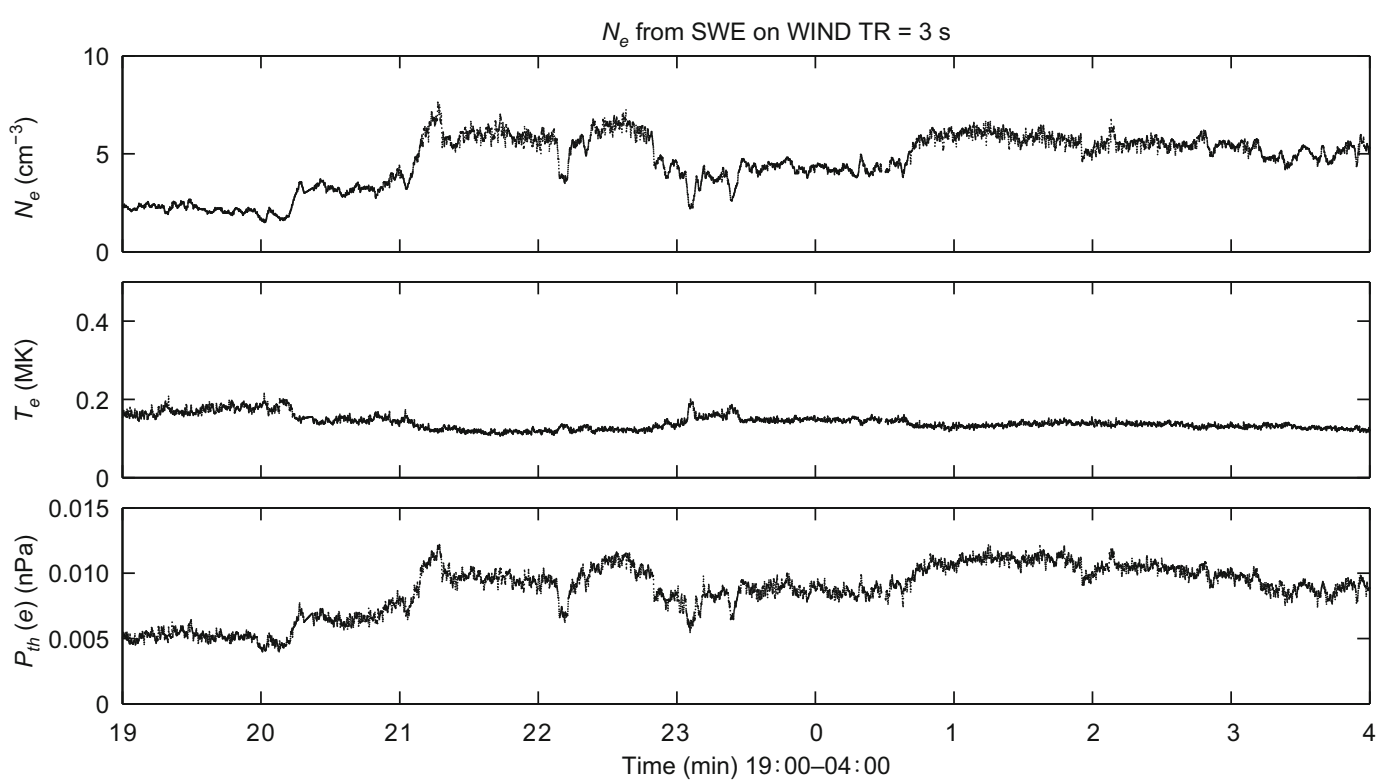

Figure 2 An overview of the measurements from SWE on WIND between 19:00 April 5th and 04:00 April 6th, 2001 UT. The first panel shows the time variation of electron density. The second panel shows the electron temperature and the third panel shows the electron thermal pressure. The measurements are shown in the figure as data points.

shown in Figures 1 and 2. The magnetic field stays steady between 5 and 8 nT, except for in 21:00-23:00 when the magnetic field falls down to almost $0 \mathrm{nT}$ twice, shown in Figure 3. The plasma thermal pressure is between 0.01 and $0.03 \mathrm{nPa}$, and the magnetic pressure varies between 0 and $0.03 \mathrm{nPa}$.
The total pressure is stable around $0.04 \mathrm{nPa}$, and the plasma $\beta$ is mostly less than 5, except for inside 21:00-23:00 soaring to 10 twice, shown in Figure 4.

In this period, we notice a 2-hour temporal interval from 23:00 April 5th to 01:00 April 6th in a rather steady state, 


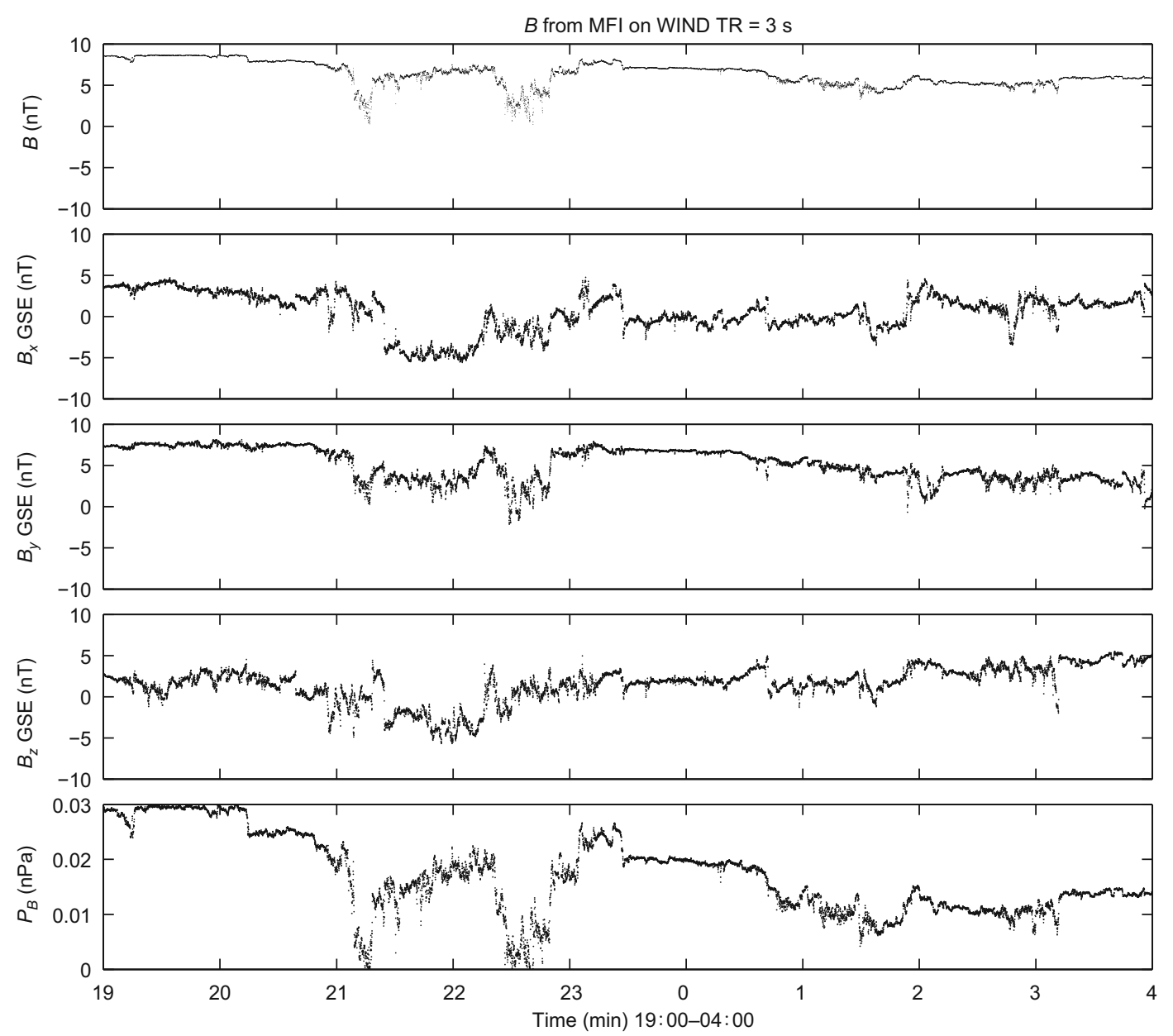

Figure 3 An overview of the measurements from MFI on WIND between 19:00 April 5th and 04:00 April 6th, 2001 UT. The four panels show the magnitude and the $x, y, z$ components of the magnetic field in GSE coordinate. The measurements are shown in the figure as data points.

with stable total pressure, plasma $\beta$, magnetic field strength, and solar wind velocity. In Figure 5, the electron density, the proton density, the total magnetic field strength, the plasma kinetic pressure, the magnetic pressure and the total pressure of the two hours are shown from the top panel to the bottom panel. The $P_{t h}$ and $P_{B}$ vary oppositely when the total pressure stays steady. Obviously, the anti-correlation between $P_{t h}$ and $P_{B}$ appears from time to time. Thus, we suppose pressurebalanced structures may be embedded in this period's solar wind. To investigate the relation of $P_{t h}$ and $P_{B}$, as well as that of $N_{e}$ and $B$, we apply the wavelet cross coherence analysis. In this work, we have studied the cross spectra of both coherence coefficient and the coherent angle.

First, we adopt the wavelet cross coherence analysis to reveal the detailed anti-correlation between $P_{t h}$ and $P_{B}$. In Figure 6 , we calculate the coefficient and coherent angle spectrum of $P_{t h}$ and $P_{B}$, using the wavelet transformation package from [23]. Figure 6 shows the wavelet cross-coherence spectra between $P_{t h}$ and $P_{B}$ with a period ranging from 10 to $1000 \mathrm{~s}$ and a temporal interval between 23:00 and 01:00 UT. The left panel shows the spectrum of the coherence coefficient, with its color bar ranging from 0 to 1 as blue to red, and the right panel is the spectrum of the absolute coher- ence phase angle, with its color bar ranging from 0 to $\pi$ as blue to red. In both panels, the black contours represent the coherence coefficient to be 0.8 . Thus, the red region inside the black contours in the left panel indicates where the crosscoherence coefficient is larger than 0.8 . For easy comparison, these contours are repeated in the right panel. Since the red region in the right panel indicates where the cross-coherence absolute phase angle is close to $\pi$ (i.e. $180^{\circ}$ ), by simultaneous inspection of both panels, we can clearly identify that the red region limited by the contours has a phase angle of $\pi$ or $-\pi$, which means that here the plasma kinetic pressure and magnetic pressure are strongly anti-correlated. This negative correlation appears in the large scales over the whole time period considered in Figure 6, but it becomes more intermittent with shorter duration, as the scale decreases (from the bottom to top of the panel). Thus Figure 6 indicates an increasing number of PBSs as temporal scale decreases. Besides, it should be mentioned that the unshadowed region in Figure 6 is the 0.95 confidential region of wavelet cross coherence analysis.

We could identify several discrete temporal intervals in which the $P_{t h}$ and $P_{B}$ are anti-correlated. As the scale shortens, the length of the temporal interval decreases. When the period (temporal scale) is larger than $1000 \mathrm{~s}$, the $P_{t h}$ and $P_{B}$ 

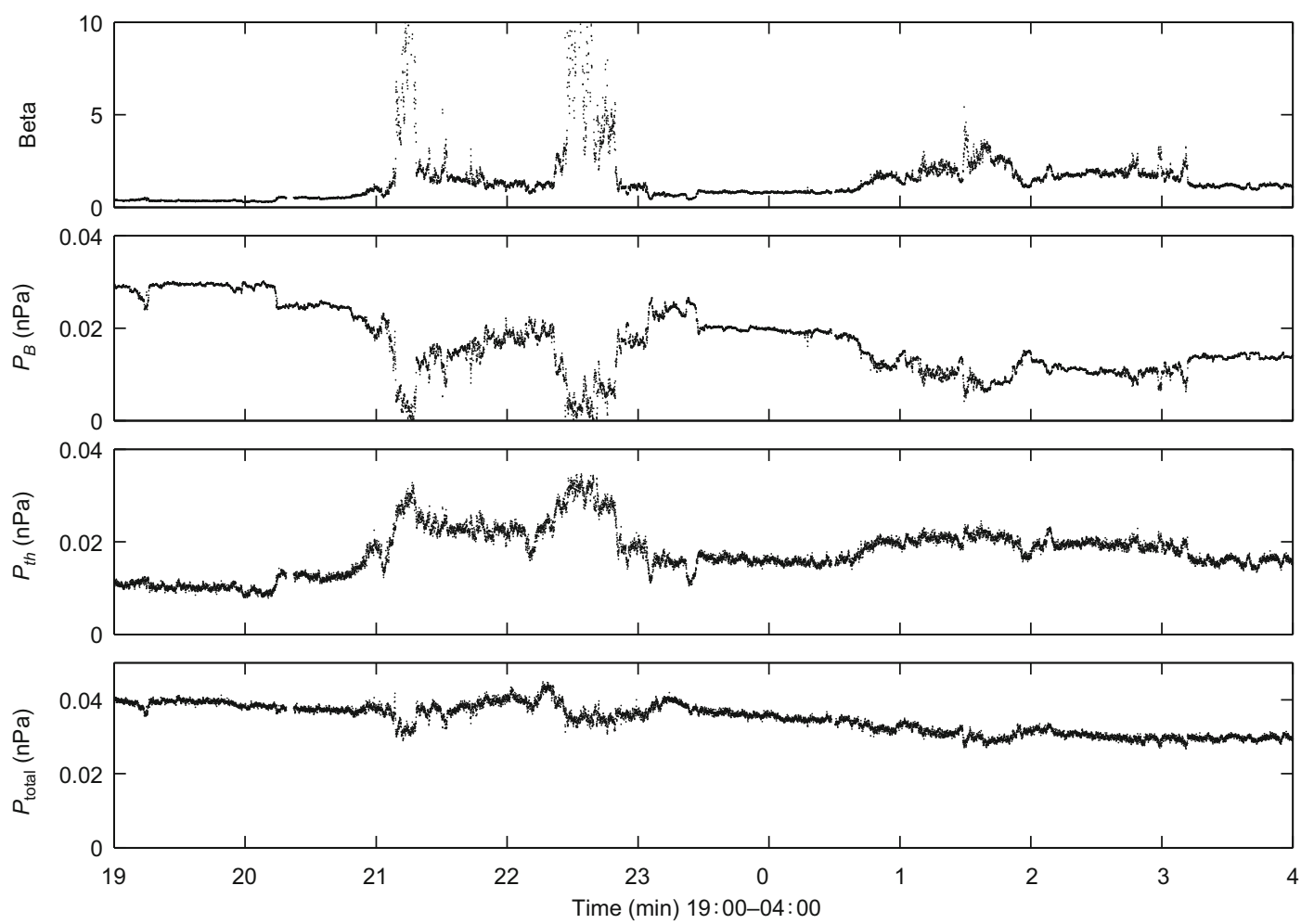

Figure 4 An overview of plasma pressure, magnetic pressure, total pressure and plasma beta between 19:00 April 5th and 04:00 April 6th, 2001 UT. The four panels show the plasma $\beta$, the plasma thermal pressure $P_{t h}$, magnetic pressure $P_{B}$, and the total pressure $P_{\text {total }}$. The measurements are shown in the figure as data points.

are anti-correlated in the whole time section from 23:00 to 01:00, showing as a red belt in the bottom of both panels of Figure 6. When the period is about $200 \mathrm{~s}$, the $P_{t h}$ and $P_{B}$ are anti-correlated between 23:40 and 23:50, 23:55 and $00: 10$, and also possibly from at least $23: 05$ to $23: 12$, for the beginning of the whole temporal interval is outside the confidential region. In general, the anti-correlation with scale of $200 \mathrm{~s}$ appears in several 10-min temporal intervals. As the period decreases to shorter than $100 \mathrm{~s}$, we notice that between 23:25 and 23:26, 23:26 and 23:27, 00:10 and 00:11, 00:19 and 00:21, and 00:48 and 00:51 the $P_{t h}$ and $P_{B}$ are anti-correlated. To sum up, the anti-correlation with scale less than $100 \mathrm{~s}$ appears in several 1-min temporal intervals.

Furthermore, we calculate the coefficient and coherent angle spectra of $N_{e}$ and $B$, using the wavelet transformation package from [23] as well. Figure 7 shows the wavelet crosscoherence spectrum between $N_{e}$ and $B$ with a period ranging from 10 to $1000 \mathrm{~s}$ and a temporal interval between 23:00 and 01:00 UT. The same as in Figure 6, the left panel shows the spectrum of the coherence coefficient, with its color bar ranging from 0 to 1 as blue to red, and the right panel is the spectrum of the absolute coherence phase angle, with its color bar ranging from 0 to $\pi$ as blue to red. To compare the information of the coefficient and the coherence angle, the black contours in both panels indicate the coefficient to be 0.8 .

We could identify some anti-correlation regions of $N_{e}$ and $B$ distributed in the whole temporal interval from 23:00 to 01:00. Compared to Figure 6, we notice that the number of anti-correlation regions of $N_{e}$ and $B$ is less than that of $P_{t h}$ and $P_{B}$. From Figure 7, we observe that with scale of $200 \mathrm{~s}$, between 23:43 and 23:47, and 23:55 and 00:08 the $N_{e}$ and $B$ are anti-correlated. With scale of $100 \mathrm{~s}, N_{e}$ and $B$ are anticorrelated between 00:48 and 00:50. And with scale of $40 \mathrm{~s}$, $N_{e}$ and $B$ are anti-correlated between 00:18 and 00:19. Obviously, these temporal intervals are just the among the temporal intervals when $P_{t h}$ and $P_{B}$ are of anti-correlation, though for $N_{e}$ and $B$ the anti-correlation lasts for shorter time than $P_{t h}$ and $P_{B}$.

Figures 6 and 7 show the wavelet cross-coherence spectra of $P_{t h}-P_{B}$ and $N_{e}-B$, respectively, with different temporal scales from $10 \mathrm{~s}$ to $10^{3} \mathrm{~s}$. According to the time variation of $N_{e}-B$ and $P_{t h}-P_{B}$, and the steady total pressure shown in Figure 5, we believe the solar wind we study is composed of pressure-balanced structures over multi-scale. It indicates that the PBSs also exist on small temporal scales (less than one hour), not only on large temporal scales (longer than one hour) in the solar wind turbulence. Since the anti-correlation between $P_{t h}$ and $P_{B}$ stays continuously over multi-scale in several temporal intervals, shown in Figure 5, we suppose this phenomenon may indicate the small PBSs embedded in the larger ones.

\section{Discussion}

For the first time, we observe the anti-correlation between the fluctuations of $P_{t h}$ and $P_{B}$ from their time variation and their 


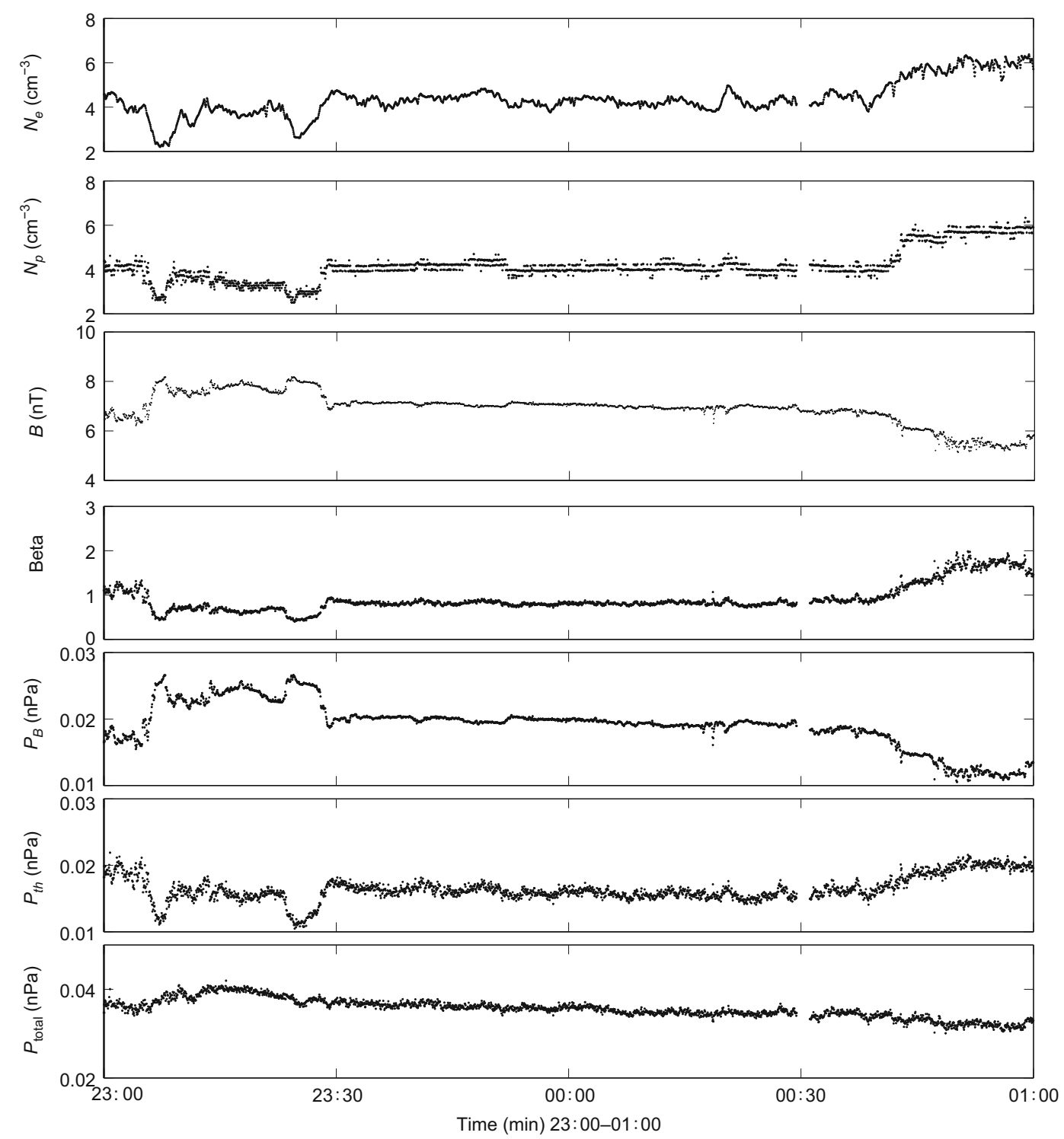

Figure 5 The time variation of the electron density, proton density, magnetic field magnitude, plasma thermal pressure, magnetic pressure, plasma $\beta$ and total pressure from 23:00 April 5th to 01:00 April 6th. From the top to the bottom panel, the electron density, the proton density, the magnetic field magnitude, the plasma $\beta$, the plasma thermal pressure, the magnetic pressure and the total pressure are displayed respectively. The measurements are shown in the figure as data points.
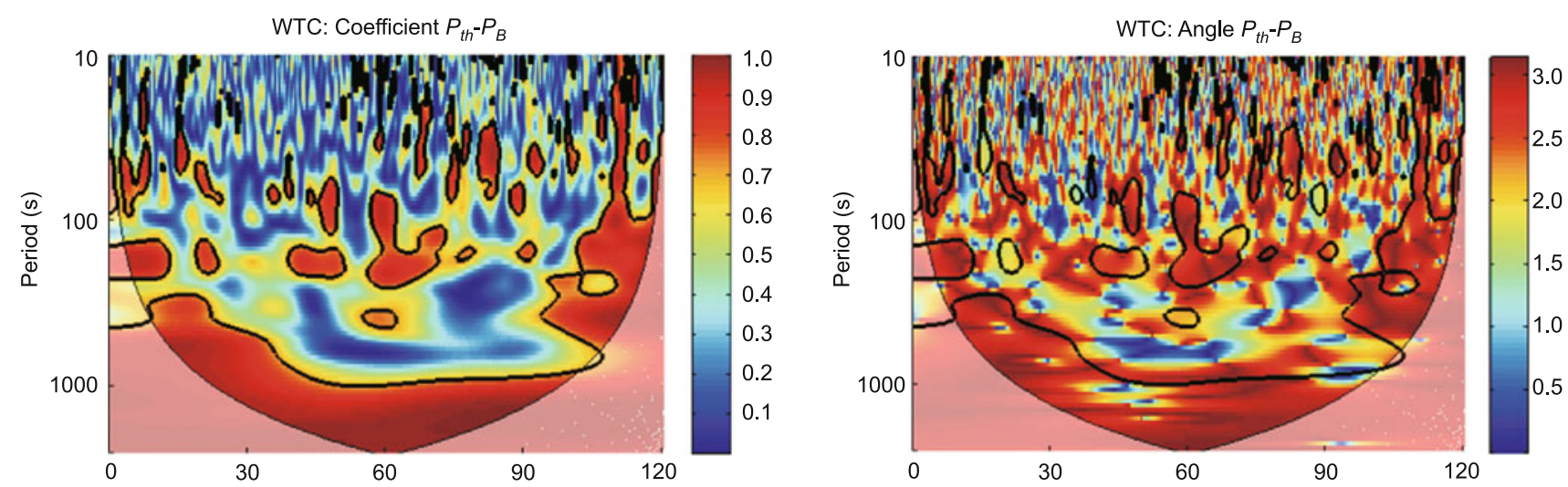

Figure 6 The left panel shows the wavelet cross coherence coefficient spectrum between $P_{t h}$ and $P_{B}$ in the temporal interval between 23:00 April 5th and 01:00 April 6th, 2001. The color represents the correlation coefficient and the black contour marks the coefficient to be 0.8. In the right panel, the wavelet cross coherence angle spectrum of $P_{t h}$ and $P_{B}$ is shown. The color represents the angle and the black contour marks the coefficient to be 0.8 . 

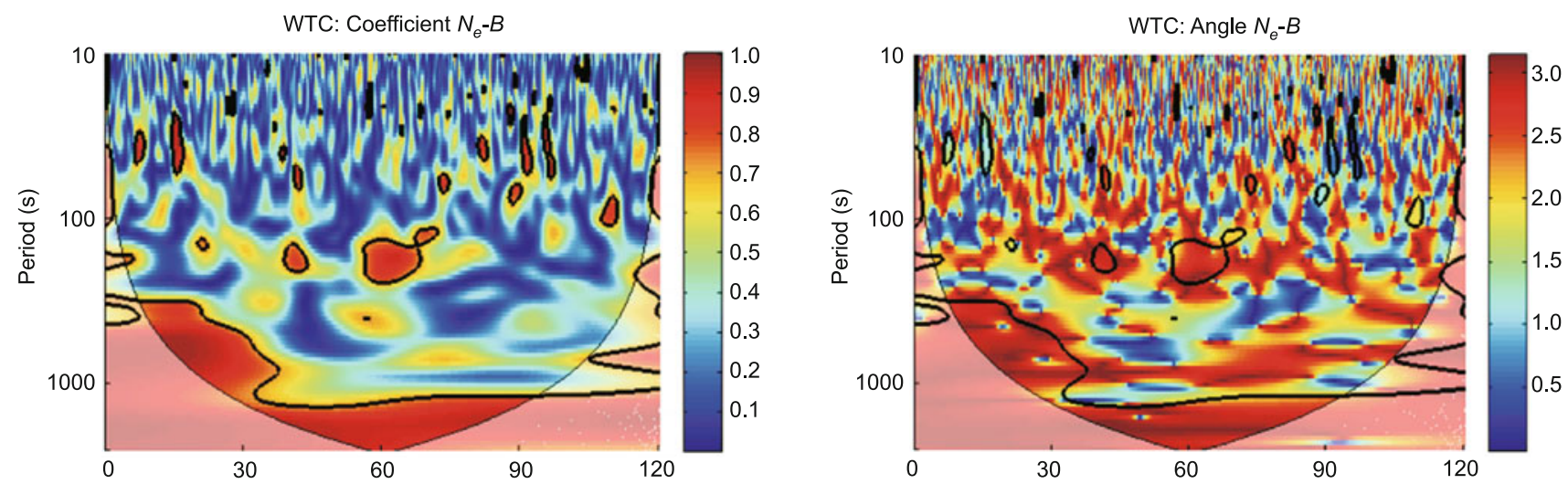

Figure 7 The left panel shows the wavelet cross coherence coefficient spectrum between $N_{e}$ and $B$ in the temporal interval between 23:00 April 5th and 01:00 April 6th, 2001. The color represents the coefficient and the black contour marks the coefficient to be 0.8 . In the right panel, the wavelet cross coherence angle spectrum of $N_{e}$ and $B$ is shown. The color represents the angle and the black contour marks the coefficient to be 0.8 .

wavelet cross-coherence spectra over a large range of scales from one hour down to ten seconds during the same solar wind period. Our observation for the first time offers the direct evidence to support the existence of small scale (lasting time less than one hour) pressure-balanced structures in the solar wind. If we calculate the spacial scale of the pressurebalanced structures simply by the solar wind velocity and the duration of the anti-correlation of $P_{t h}$ and $P_{B}$, the multi-scale PBSs observed in our study are from 5000 to $500000 \mathrm{~km}$.

To compare the correlation between $N_{e}$ and $B$ with that between $P_{t h}$ and $P_{B}$ in the pressure-balanced structures, we also investigate the relation between $N_{e}$ and $B$ by the wavelet cross-coherence spectrum. As a result, we observe a similar pattern of the $P_{t h}-P_{B}$ and $N_{e}-B$ spectra. In detail, the temporal intervals of anti-correlation for $N_{e}-B$ are among that of $P_{t h}-P_{B}$, but with shorter lasting time.

Previously, Yao et al. [16] discussed the producing mechanism of PBSs by the electron density fluctuation power spectra density (PSD), and from the PSD's slope as $-5 / 3$ speculated that the PBSs may be dominated by the cascading of Alfvénic fluctuation. In order to explain the PBSs observed by WIND in our study, we decide to compare our electron fluctuation PSD with that of [16]. In the comparison displayed in Figure 8, we observe the similar trend of the $N_{e}$ fluctuation spectra measured by WIND and Cluster on April 5th, 2001 at $1 \mathrm{AU}$. The power density spectrum (PSD) of $N_{e}$ measured by WIND covers the frequency from 0.001 to $0.1 \mathrm{~Hz}$, showing a slope of -1.97 . While the PSD of $N_{e}$ measured by Cluster 1 [16] covers the range from 0.01 to $2.5 \mathrm{~Hz}$, having a slope of -1.51 . The slopes close to $-5 / 3$ suggest that the fluctuation of $N_{e}$ is controlled by the Alfvénic cascading. Considering the time when Cluster and WIND detected the PBSs is almost the same, with the similar solar wind velocity and the similar magnetic field strength, we tend to believe the PBSs detected by Cluster and WIND have the same origin.

One of the physical explanations for the existence of the multi-scale PBSs in the solar wind at 1 AU could be the outward propagating flux tubes from the solar corona. Thieme et al. [3] suggested that the large scale PBSs, with temporal

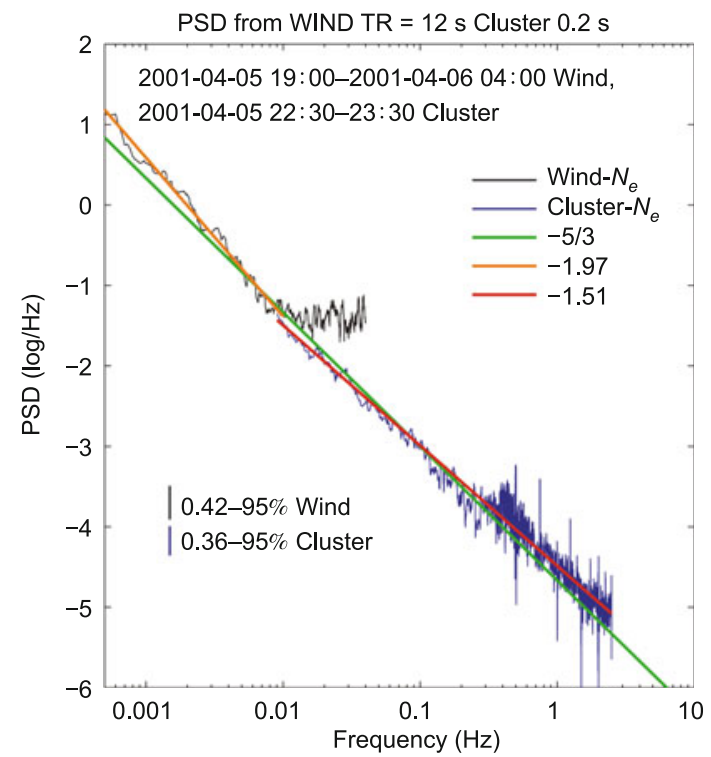

Figure 8 Relative fluctuation spectra of $N_{e}$ measured by WIND and Cluster at $1 \mathrm{AU}$ on April 5th and 6th, 2001. The $x$ axis is frequency and the $y$ axis is the logarithmical value of the normalized power spectrum. The spectra are fitted by the least-mean-square method, and the slopes are given in the labels, which are close to $-5 / 3$. Besides, in the application of the multitaper method the number of data tapers is set to be 12 .

scale larger than 1 hour or spatial scale larger than $10^{6} \mathrm{~km}$, may originate from the network with size of $10000 \mathrm{~km}$. Considering the smallest PBSs observed in our study are about $5000 \mathrm{~km}$ in size, which are less than one percent of the large scale ones, thus the flux tubes forming the small scale PBSs have to be less than $100 \mathrm{~km}$ 's size on the sun. However, such a small size structure in the solar atmosphere touches the resolution limit of the optical observation. A possible corresponding magnetic structure is the magnetic element, though the magnetic element itself still waits for observational confirmation.

Besides, the in situ slow magnetosonic waves and the mirror mode waves could also contribute to the formation of PBSs, as they both have the anti-correlation between $N_{e}$ and $B$ as a feature [24]. Above all, we could not determine the 
true producing process of these multi-scale PBSs so far. And further investigation is required to reveal more physical nature of these small scale PBSs.

The data in this work are from CDA web supported by NASA. Yao's work was supported by the Fundamental Research Funds for the Central Universities (2011 YYL127) and Tu's work was supported by the National Natural Science Foundation of China (40874090, 40931055 and 40890162).

1 Tu C Y, Marsch E. MHD structures and turbulence in the solar wind: Observations and theories. Space Sci Rev, 1995, 73: 1-210

2 Burlaga L F, Ogilvie K W. Magnetic and thermal pressure in the solar wind. Solar Phys, 1970, 15: 61-71

3 Thieme K M, Marsch E, Schwenn R. Spatial structures in high-speed streams as signatures of fine structures in coronal holes. Ann Geophys, 1990, 8: 713-723

4 Marsch E, Tu C Y. Correlations between the fluctuations of pressure, density, temperature and magnetic field in the solar wind. Ann Geophys, 1993, 11: 659-677

5 Tu C Y, Marsch E. On the nature of compressive fluctuations in the solar wind. J Geophys Res, 1994, 99: 21481-21509

6 McComas D J, Barraclough B L, Gosling J T, et al. Structures in the polar solar wind: Plasma and field observations from Ulysses. J Geophys Res, 1995, 100: 19893-19902

7 Reisenfeld D B, McComas D J, Steinberg J T. Evidence of a solar origin for pressure balance structures in the high-latitude solar wind. Geophys Res Lett, 1999, 26: 1805-1808

8 Bavassano B, Pietropaolo E, Bruno R. Compressive fluctuations in highlatitude solar wind. Ann Geophys, 2004, 22: 689-696

9 Zhao H, Zong Q G, Wei Y, et al. Influence of solar wind dynamic pressure on geomagnetic Dst index during various magnetic storms. Sci China Tech Sci, 2011, 54: 1445-1454

10 Wei Y, Zong Q G, Pu Z Y, et al. Enhanced anti-sunward flow near local noon during a period of horizontal Imf and high solar wind velocity $V_{Y}$. Chin Sci Bull, 2011, 56: 1117-1122
11 Zong Q G, Wang Y F, Yuan C J, et al. Fast acceleration of killer electrons and energetic ions by interplanetary shock stimulated Ulf waves in the inner magnetosphere. Chin Sci Bull, 2011, 56: 1188-1201

12 Goldstein B, Siscoe G L. Spectra and cross spectra of solar wind parameters from Mariner 5. NASA Special Publication, 1972, 308: 506-516

13 Feng Y Y, Zhang Y T, Zhao H, et al. Solar wind electrons reflected by lunar electric and magnetic fields. Sci China Earth Sci, 2011, 54: 1796-1800

14 Kellogg P J, Horbury T S. Rapid density fluctuations in the solar wind. Ann Geophys, 2005, 23: 3765-3773

15 Howes G G, Bale S D, Klein K G, et al. The slow-mode nature of compressible wave power in solar wind turbulence. arXiv: $1106.4327 \mathrm{v} 1$

16 Yao S, He J S, Marsch E, et al. Multi-scale anti-correlation between electron density and magnetic field strength in the solar wind. Astrophys J, 2011, 728: 146

17 Ogilvie K W, Desch M D. The WIND spacecraft and its early scientific results. Adv Space Sci, 1997, 20: 559-568

18 Lin R P, Anderson K A, Ashford S, et al. The three dimensional plasma and energetic particle investigation for the wind spacecraft. Space Sci Rev, 1995, 71: 125-153

19 Ogilvie K W, Chornay D J, Frizenreiter R J, et al. SWE, a comprehensive plasma instrument for the WIND spacecraft. Space Sci Rev, 1995, 71: $55-77$

20 Lepping R P, Acuna M H, Burlaga L F, et al. The wind magnetic field investigation. Space Sci Rev, 1995, 71: 207-229

21 Percival D B, Walden A T. Spectral Analysis for Physical Applications - Multitaper and Conventional Univariate Techniques. Cambridge: Cambridge University Press, 1993

22 Eastwood J P, Phan T D, Bale S D, et al. Observations of turbulence generated by magnetic reconnection. Phys Rev Lett, 2009, 102: 035001

23 Grinsted A, Moore J C, Jevrejeva S. Application of the cross wavelet transform and wavelet coherence to geophysical time series. Nonlinear Proc Geophys, 2004, 11: 561-566

24 Song P, Russell C T, Thomsen M F. Waves in the inner magnetosheath: A case study. Geophys Res Lett, 1992, 19: 2191-2194

Open Access This article is distributed under the terms of the Creative Commons Attribution License which permits any use, distribution, and reproduction in any medium, provided the original author(s) and source are credited. 